TAIWANESE JOURNAL OF MATHEMATICS

Vol. 12, No. 4, pp. 991-996, July 2008

This paper is available online at http://www.tjm.nsysu.edu.tw/

\title{
A CLASS OF LYM ORDERS IN DIVISOR LATTICES
}

\author{
Yi Wang and Yeong-Nan Yeh
}

Dedicated to Professor Ko-Wei Lih on the occasion of his 60th birthday.

\begin{abstract}
We present a new class of LYM orders, which generalizes Lih's result and is a common generalization of Griggs' result and a result of West, Harper and Daykin.
\end{abstract}

\section{INTRODUCTION}

A partially ordered set (or poset) is a set equipped with a reflexive, antisymmetric, and transitive relation. A poset $P$ is ranked if there is a rank function $r: P \rightarrow \mathbb{N}$ such that $r(x)=0$ if $x$ is a minimal element of $P$ and $r(z)=r(y)+1$ if $z$ covers $y$ in $P$. We call $r(x)$ the rank of $x$. The rank of $P$ is the maximum value of $r(x)$ taken over all $x \in P$. Let $P_{i}$ denote the set of elements of rank $i$ in $P$. Its cardinality $\left|P_{i}\right|$ is called the ith Whitney number of $P$. We say that $P$ is $L C$ if the Whitney numbers of $P$ form a log-concave sequence, that is,

$$
\left|P_{i}\right|^{2} \geq\left|P_{i-1}\right| \cdot\left|P_{i+1}\right|
$$

for all $i>0$. An antichain is a subset of pairwise incomparable elements of $P$. We say that $P$ has the Sperner property if the maximum size of an antichain in $P$ equals the largest Whitney number of $P$. We say that $P$ has the LYM property if

$$
\sum_{i}\left|A \cap P_{i}\right| /\left|P_{i}\right| \leq 1
$$

for every antichain $A$ of $P$. It is well known that the LYM property implies the Sperner property ([5]).

Received December 17, 2007, Accepted January 7, 2008.

Communicated by Hung-Lin Fu.

2000 Mathematics Subject Classification: Primary 05D05; Secondary 06A07.

Key words and phrases: Posets, Divisor lattices, LYM orders.

Supported partially by NSFC Grant No. 10771027. 
The subset lattice or the Boollean lattice $B_{n}$ is the poset of all subsets of an $n$-element set, ordered by inclusion. In 1928, Sperner [13] showed, in current terminology, that the subset lattice has the Sperner property. In 1967, Rota [12] made a famous conjecture that the partition lattice has the Sperner property. Although the conjecture was shown to be invalid in general by Canfield [1] in 1978, efforts to prove analogues of Sperner's theorem for other posets have led to the emergence of an entire theory (see [4] for details). In 1980, Lih [11] discovered a generalization of Sperner's theorem. Let $X=\{1,2, \ldots, n\}$ be an $n$-element set and $Y$ a subset of $k$ elements of $X$ where $k \leq n$. Let $C(n, k)$ be the collection of all subsets of $X$ which intersect $Y$, ordered by inclusion. Lih showed that $C(n, k)$ has the Sperner property. Griggs [6] further showed, among other things, that $C(n, k)$ has the LYM property before long. He also generalized this result as follows.

Theorem 1. ([6]). Let $X=\{1,2, \ldots, n\}$ be partitioned into parts $X_{1}, X_{2}$, $\ldots, X_{r}$. Suppose that $I_{i} \subseteq\left\{0,1, \ldots,\left|X_{i}\right|\right\}$ is an arithmetic progression for each $i$. Then

$$
P=\left\{Z \subseteq X:\left|Z \cap X_{i}\right| \in I_{i}, 1 \leq i \leq r\right\},
$$

ordered by inclusion, is LYM and LC.

On the other hand, West, Harper and Daykin [16] gave a different generalization of Lih's result.

Theorem 2. ([16]). Let $C_{1} \subset C_{2} \subset \cdots \subset C_{s}$ be a chain of subsets of $X=\{1,2, \ldots, n\}$. Suppose that $\left\{a_{i}\right\}$ and $\left\{b_{i}\right\}$ are two nondecreasing sequences with $a_{i} \leq b_{i}$ for $1 \leq i \leq s$. Then

$$
P=\left\{Z \subseteq X: a_{i} \leq\left|Z \cap C_{i}\right| \leq b_{i}, 1 \leq i \leq s\right\},
$$

ordered by inclusion, is LYM and LC.

They also hoped to find out a common generalization of their result and that of Griggs. Indeed, there are similarities in the statements and the proofs of Theorem 1 and Theorem 2. In this note we broaden these results to the divisor lattice and give a common generalization.

Let $n=p_{1}^{e_{1}} p_{2}^{e_{2}} \cdots p_{t}^{e_{t}}$ be a positive integer, where the $p_{i}$ are distinct primes and $e_{i} \in \mathbb{N}$. The divisor lattice $D(n)$ is the poset of all (positive) divisors of $n$, ordered by divisibility. As usual, let $\sigma(n)=e_{1}+e_{2}+\cdots+e_{t}$ denote the number of prime divisors of $n$ counted according to multiplicity. Then $D(n)$ is a ranked poset with the rank function $\sigma$. Clearly, $D(n)$ reduces to $B_{n}$ when $n$ is squarefree. Denote by $(m, k)$ the largest common divisor of two positive integers $m, k$ and replace $\sigma((m, k))$ by $\sigma(m, k)$. Given two nonnegative integers $a \leq b$, denote $[a, b]=\{i \in \mathbb{N}: a \leq i \leq b\}$. Our main result is the following. 
Theorem 3. Let $n=n_{1} n_{2} \cdots n_{r}$ where $n_{i}$ are positive integers of pairwise coprime. Suppose that $I_{i} \subseteq\left[0, \sigma\left(n_{i}\right)\right]$ is an arithmetic progression and $J_{i}=\left[a_{i}, b_{i}\right]$ where $a_{i} \leq b_{i}$ for each $i$. Then

$$
P=\left\{m \in D(n): \sigma\left(m, n_{j}\right) \in I_{j} \text { and } \sum_{i=1}^{j} \sigma\left(m, n_{i}\right) \in J_{j} \text { for } 1 \leq j \leq r\right\},
$$

ordered by divisibility, is $L Y M$ and $L C$.

When $n$ is square-free, the corresponding result is the following.

Corollary 1. Suppose that $X=\{1,2, \ldots, n\}$ is partitioned into parts $X_{1}, X_{2}$, $\ldots, X_{r}$. Let $I_{i} \subseteq\left[0,\left|X_{i}\right|\right]$ be an arithmetic progression and $J_{i}=\left[a_{i}, b_{i}\right]$ where $a_{i} \leq b_{i}$ for each $i$. Then

$$
P=\left\{Z \subseteq X:\left|Z \cap X_{j}\right| \in I_{j} \text { and } \sum_{i=1}^{j}\left|Z \cap X_{i}\right| \in J_{j} \text { for } 1 \leq j \leq r\right\},
$$

ordered by inclusion, is $L Y M$ and $L C$.

It is not difficult to see that Theorem 1 and 2 follow immediately from Corollary 1 . In fact, we can obtain Theorem 1 by putting each $J_{i}=[0, n]$ in Corollary 1 . On the other hand, suppose that $C_{1} \subset C_{2} \subset \cdots \subset C_{s}$ is a chain of subsets of $X$. Let

$$
X_{1}=C_{1}, X_{2}=C_{2} \backslash C_{1}, \ldots, X_{s}=C_{s} \backslash C_{s-1}, X_{s+1}=X \backslash C_{s} .
$$

Then $X_{1}, X_{2}, \ldots, X_{s}, X_{s+1}$ is a partition of $X$ and $C_{i}=X_{1} \cup X_{2} \cup \cdots \cup X_{i}$ $(1 \leq i \leq s)$. We obtain Theorem 2 by putting $J_{s+1}=[0, n]$ and $I_{i}=\left[0,\left|X_{i}\right|\right]$ $(1 \leq i \leq s+1)$ in Corollary 1 .

\section{Proof of Theorem 3}

We use the product theorem for LYM posets to prove Theorem 3. The (direct) product $Q_{1} \times Q_{2}$ of two posets $Q_{1}$ and $Q_{2}$ is defined to be the set of all pairs $\left(q_{1}, q_{2}\right), q_{1} \in Q_{1}, q_{2} \in Q_{2}$, with the order given by $\left(q_{1}, q_{2}\right) \leq\left(q_{1}^{\prime}, q_{2}^{\prime}\right)$ if and only if $q_{1} \leq q_{1}^{\prime}$ in $Q_{1}$ and $q_{2} \leq q_{2}^{\prime}$ in $Q_{2}$. Furthermore, the product of two ranked posets $Q_{1}$ and $Q_{2}$ is defined to be the poset together with the rank function $r$ given by $r\left(q_{1}, q_{2}\right)=r_{1}\left(q_{1}\right)+r_{2}\left(q_{2}\right)$, where $r_{1}$ and $r_{2}$ are the rank functions of $Q_{1}$ and $Q_{2}$, respectively. The product of two LYM posets $P$ and $Q$ may not be LYM in general, but it will be true if $P$ and $Q$ are LC also. The following result is discovered by Harper [7] and later independently by Hsieh and Kleitman [10]. 
Product Theorem. If two posets $Q_{1}, Q_{2}$ are both $L Y M$ and $L C$, then so is their product poset $Q_{1} \times Q_{2}$.

A subposet of a poset $Q$ is a subset of $Q$ whose elements are ordered as in $Q$. Let $Q=\bigcup_{i=0}^{n} Q_{i}$ be a poset of rank $n$. Given a subset $I$ of $[0, n]$, let $Q_{I}=\bigcup_{i \in I} Q_{i}$ be the subposet of $Q$ induced by $I$. Clearly, an antichain of $Q_{I}$ is also antichain of $Q$. It follows that if the poset $Q$ is LYM, then so is the subposet $Q_{I}$.

Let $\left\{W_{i}\right\}_{i=0}^{n}$ be a log-concave sequence of positive numbers. Then the sequence $\left\{W_{i} / W_{i-1}\right\}_{i=1}^{n}$ is nonincreasing. Thus $W_{j} / W_{j-1} \geq W_{k} / W_{k-1}$ for $j \leq k$, or equivalently, $W_{j} W_{k-1} \geq W_{j-1} W_{k}$. It follows that

$$
W_{i}^{2} \geq W_{i-1} W_{i+1} \geq W_{i-2} W_{i+2} \geq \cdots \geq W_{i-d} W_{i+d} .
$$

Let $I=\{a, a+d, a+2 d, \ldots, a+m d\}$ be an arithmetic progression in the closed interval $[0, n]$. Then the inequality (2.1) implies that the subsequence $\left\{W_{i}\right\}_{i \in I}$ is log-concave.

From the above discussion, we can conclude the following.

Lemma 1. Let $Q$ be a ranked poset of rank $n$ and let $I$ be an arithmetic progression in the closed interval $[0, n]$. If $Q$ is $L Y M$ and $L C$, then so is the subposet $Q_{I}$ induced by $I$.

We now prove Theorem 3 .

Proof of Theorem 3. We proceed by induction on $r$. If $r=1$, then

$$
P=\{m \in D(n): \sigma(m, n) \in I \cap J\},
$$

where $I \subseteq[1, \sigma(n)]$ is an arithmetic progression and $J=[a, b]$. Clearly, $I \cap J$ is still an arithmetic progression. Note that $P$ consists of those elements of $D(n)$ with rank in $I \cap J$ and it is also well known that $D(n)$ is LYM and LC ([3]). Hence the subposet $P$ of $D(n)$ is LYM and LC by Lemma 1 .

Suppose next that $r>1$. Consider the following two posets:

$$
P_{1}=\left\{m \in D\left(n_{r}\right): \sigma\left(m, n_{r}\right) \in I_{r}\right\}
$$

and

$$
\begin{aligned}
P_{2}=\left\{m \in D\left(n_{1} \cdots n_{r-1}\right):\right. & \sigma\left(m, n_{j}\right) \in I_{j} \text { and } \sum_{i=1}^{j} \sigma\left(m, n_{i}\right) \in J_{j} \\
& \text { for } 1 \leq j \leq r-1\} .
\end{aligned}
$$

By the induction hypotheses and Lemma 1 , both $P_{1}$ and $P_{2}$ are LYM and LC. So $P_{1} \times P_{2}$ is also LYM and LC by the Product Theorem. Note that $P_{1} \times P_{2}$ is 
isomorphic to the subposet of $D(n)$

$$
\begin{aligned}
Q=\{m \in D(n): & \sigma\left(m, n_{j}\right) \in I_{j} \text { for } 1 \leq j \leq r \text { and } \\
& \left.\sum_{i=1}^{j} \sigma\left(m, n_{i}\right) \in J_{j} \text { for } 1 \leq j \leq r-1\right\}
\end{aligned}
$$

and that $P$ is the subposet $Q_{J_{r}}$ of $Q$ induced by $J_{r}$. Hence $P$ is LYM and LC by Lemma 1. This completes the proof of Theorem 3.

\section{REMARKS}

Let $F$ be a collection of $t$-subsets of $X=\{1, \cdots, n\}$. Consider the filter generated by $F$ :

$$
P(F)=\{Y \subseteq X: Y \supseteq A \text { for some } A \in F\},
$$

which is a subposet of the Boolean lattice $B_{n}$. Lih [11] conjectured that $P(F)$ has the Sperner property. The case $t=0$ is just the classical Sperner theorem and the case $t=1$ is Lih's result about $C(n, k)$. However, Zhu [18] found counterexamples to the conjecture with $t>n / 2$. Griggs [6] showed that the conjecture fails for $t=4$ and Zha [17] constructed counterexamples for all $t \geq 4$ and $n \geq 2 t-1$. Horrocks $[8,9]$ gave a graph-theoretical interpretation for the $t=2$ conjecture and left 116 exceptional graphs in his proof. Cheng and Lih [2] carried on further with Horrocks's reduction method to reduce the number of exceptional graphs and gave a complete proof for the $t=2$ conjecture. The conjecture remains open for $t=3$. An interesting problem is to consider analogue of Lih's conjecture for the divisor lattices and other posets. We also refer the reader to $[14,15]$ for a subspace lattice analogue of Lih's poset $C(n, k)$.

\section{REFERENCES}

1. E. R. Canfield, On a problem of Rota, Adv. Math., 29 (1978), 1-10.

2. S.-E. Cheng and K.-W. Lih, An improvement on a Spernerity proof of Horrocks, Theoret. Comput. Sci., 263 (2001), 355-377.

3. G. F. Clements, Antichains in the set of subsets of a multiset, Discrete Math., 48 (1984), 23-45.

4. K. Engel, Sperner Theory, Cambridge University Press, Cambridge, 1997.

5. C. Greene and D. J. Kleitman, Proof techniques in the theory of finite sets, in: Studies in Combinatorics, MAA Stud. Math., 17, Math. Assoc. America, Washington, D.C., 1978, pp. 22-79. 
6. J. R. Griggs, Collections of subsets with the Sperner property, Trans. Amer. Math. Soc., 269 (1982), 575-591.

7. L. H. Harper, The morphology of partially ordered sets, J. Combin. Theory Ser. A, 17 (1974), 44-58.

8. D. G. C. Horrocks, Nested chain partitions of Hamiltonian filters, J. Combin. Theory Ser. $A, 81$ (1998), 176-189.

9. D. G. C. Horrocks, On Lih's conjecture concerning Spernerity, European J. Combin., 20 (1999), 131-148.

10. W. N. Hsieh and D. J. Kleitman, Normalized matching in direct products of partial orders, Stud. Appl. Math., 52 (1973), 285-289.

11. K.-W. Lih, Sperner families over a subset, J. Combin. Theory Ser. A, 129 (1980), 182-185.

12. G.-C. Rota, A generalization of Sperner's theorem, J. Combin. Theory, 2 (1967), 104.

13. E. Sperner, Ein Satz über Untermengen einer endlichen Menge, Math. Z., 27 (1928), 327-330.

14. J. Wang and H. J. Zhang, Normalized matching property of a class of subspace lattices, Taiwanese J. Math., 11 (2007), 43-50.

15. Y. Wang, On a class of subspace lattices, J. Math. Res. Exposition, 19 (1999), 341-348.

16. D. B. West, L. H. Harper and D. E. Daykin, Some remarks on normalized matching, J. Combin. Theory Ser. A, 35 (1983), 301-308.

17. X. Y. Zha, On a conjecture on the Sperner property, European J. Combin., 10 (1989), 603-607.

18. Y. X. Zhu, A note on a conjecture concerning the Sperner property (in Chinese), $J$. Math. Res. Exposition, 4 (1984), 148.

\author{
Yi Wang \\ Department of Applied Mathematics, \\ Dalian University of Technology, \\ Dalian 116024, \\ P. R. China \\ E-mail: wangyi@dlut.edu.cn \\ Yeong-Nan Yeh \\ Institute of Mathematics, \\ Academia Sinica, \\ Taipei 11529, Taiwan \\ E-mail: mayeh@math.sinica.edu.tw
}

